REVISTA DE DERECHO UNED, NÚM. 27, 2021

\title{
LA REFORMA DE LA GUARDIA EUROPEA DE FRONTERAS Y COSTAS CON EL REGLAMENTO (UE) $2019 / 1896^{1}$
}

\author{
THE REFORM OF THE EUROPEAN BORDER AND COAST \\ GUARD WITH REGULATION (EU) 2019/1896
}

\section{MIGUEL ÁNGEL ESTEBAN CARRASCO²}

Doctorando de la Universidad Nacional de Educación a Distancia (UNED)

Sumario: I. Introducción. II. El fingido aumento de la capacidad de la guardia europea de fronteras y costas (gefc). II.A. Capacidad operativa. II.B. Capacidad financiera. III. El techo de la competencia compartida. III.A. El artículo 4.2 Tfue y su encaje en la versión 2.0 De la gefc. III.B. Idénticas tareas en las versiones 1.0 y 2.0 De la gefc. III.B.1. Tareas retocadas. III.B.2. Tareas existentes puestas en valor. III.B.3. Tareas auxiliares. III.B.3.A). Sistema de documentos auténticos y falsos en red (fado). III.B.3.B). Sistema europeo de información y autorización de viajes (seiav). III.B.4. Miscelánea recopiladora. IV. La potenciación en la externalización. IV.A. Gestión europea integral de fronteras. IV.A.1. Cooperación de estados miembros con terceros países. IV.A.2. Cooperación de la agencia con terceros países. IV.A.2.A). Acuerdos de estatuto. IV.A.2.B). Acuerdos de trabajo. IV.A.3. Relevan-

1 El contenido de este artículo forma parte de la investigación doctoral que realiza el autor en el programa de Derecho y Ciencias Sociales de la UNED, con el título "FRONTEX la agencia inagotablemente gris".

2 Licenciado en Derecho, Graduado en Ciencia Política y de la Administración, Graduado en Ciencias Jurídicas de las Administraciones Públicas y Máster Universitario en Derechos Humanos, todos ellos de la Universidad Nacional de Educación a Distancia (UNED). 
cia de la cooperación con terceros países. IV.A.4. Operaciones militares. IV.4.A). Operación sophia. IV.4.B). Operación irini. IV.5. Mayor externalización al mismo ritmo. $V$. Conclusiones.

Resumen: En el presente año estrenamos nuevo mandato de FRONTEX con el Reglamento UE 2019/1896 que consolida la versión 2.0 de la Guardia Europea de Fronteras y Costas. Desde la Unión Europea se anuncia un salto histórico en la gestión de fronteras llegando a afirmar la federalización de las mismas. En este estudio se analiza las novedades presentadas en esta materia por los políticos europeos y las distancias existentes entre las propuestas políticas y la realidad reglamentada. Los límites en el reparto competencial europeo vislumbran un estancamiento de la Agencia en su crecimiento cualitativo, que no cuantitativo, al chocar con la soberanía estatal. Para desentrañar el verdadero aumento de capacidades del nuevo cuerpo permanente, se comparan las tareas de los sistemas "False and Authentic Documents Online" FADO o el "Sistema Europeo de Información y Autorización de viajes" SEIAV con el texto ya derogado o se describen las operaciones SOPHIA o IRINI para tratar de conocer el futuro de la gestión de fronteras exteriores.

Palabras Clave: FRONTEX, FADO, SEIAV, SOPHIA, IRINI.

Abstract: This year we have released a new mandate for FRONTEX with EU Regulation 2019/1896 that consolidates version 2.0 of the European Border and Coast Guard. A historic leap in border management has been announced from the European Union, reaching the point of federalizing them. This study analyzes the novelties presented in this matter by European politicians and the distances between political proposals and regulated reality. The limits on the European distribution of powers show a stagnation of the Agency in its qualitative growth, rather than quantitative, when colliding with state sovereignty. To unravel the true increase in the capabilities of the new permanent body, the tasks of the FADO "False and Authentic Documents" systems or the SEIAV "European Information and Travel Authorization System" are compared with the text already repealed or SOPHIA operations are described. o IRINI to try to know the future of the management of external borders.

Key Words: frontex, fado, seiav, sophia, irini.

Recepción original: 8-5-2020

Aceptación original: 15-9-2020 


\section{INTRODUCCIÓN.}

El pasado 14 de noviembre de 2019 se publicó en el Diario Oficial de la Unión Europea el Reglamento (UE) 2019/1896 sobre la Guardia Europea de Fronteras y Costas, (en adelante GEFC). Con esta publicación, su vacatio legis de veinte días y posterior entrada en vigor, se revisa en el corto espacio de tiempo de dos años el mandato que se encargó a la entonces nueva GEFC. Nos encontramos ante la versión 2.0 de la citada Agencia, que recordemos tiene su origen y antecedentes en el Reglamento (CE) n ${ }^{\circ}$ 2007/2004 del Consejo de 26 de octubre de 2004, por el que se crea una Agencia Europea para la gestión de la cooperación operativa en las fronteras exteriores de los Estados miembros de la Unión Europea, comúnmente conocida por la denominación FRONTEX ${ }^{1}$ con la que continuará en el futuro. Esta GEFC 2.0, significa la versión FRONTEX 3.0 de la agencia como apuntan algunos autores ${ }^{2}$.

El nuevo reglamento deroga, de facto, la versión 1.0 de la $\mathrm{GEFC}^{3}$, así como el reglamento del sistema europeo de vigilancia de fronte$\mathrm{ras}^{4}$. A este último le absorbe en su totalidad y le sitúa en la sección tercera del capítulo segundo del nuevo texto.

En la redacción del reglamento de la versión 2.0, se utiliza como base el texto de la GEFC del año 2016, el cual se adapta y reforma, conforme a las necesidades del nuevo mandato que se ha pretendido establecer. La similitud entre el texto nuevo y su predecesor es muy alta. En su estructura ambos presentan cinco capítulos, diferenciándoles el número total de artículos que contienen e incrementándose los 83 anteriores en los 124 actuales, lo cual no es señal de un mayor contenido, puesto que debemos tener en cuenta que ha sido absorbido el reglamento EUROSUR.

Respecto de las novedades que este instrumento jurídico presenta y que analizaremos algunas con más detenimiento y amplitud en este artículo, podemos destacar como medida estrella, la creación de un cuerpo permanente conformado por 10.000 miembros frente

1 Considerando (3) del Reglamento (UE) 2019/1896 del Parlamento europeo y del Consejo de 13 de noviembre de 2019 sobre la Guardia Europea de Fronteras y Costas.

2 MA Acosta Sánchez, "Reglamento (UE) 2019/1896 sobre la guardia europea de fronteras y costas: ¿Frontex 3.0?”, en boletín IEEE n 16, 2019, pp. 646-666.

3 Reglamento (UE) 2016/1624 del Parlamento europeo y del Consejo de 14 de septiembre de 2016, sobre la Guardia Europea de Fronteras y Costas.

4 Reglamento (UE) 1052/2013 del Parlamento europeo y del Consejo de 22 de octubre de 2013, por el que se crea un Sistema Europeo de Vigilancia de Fronteras (EUROSUR). 
a los aproximadamente 1.300 guardias de fronteras y costas con los que cuenta la Agencia en la actualidad, esta medida junto a otras más fueron adelantadas en nota de prensa de la Comisión europea de fecha 8 de noviembre de $2019^{5}$, donde se hacía alusión como segunda medida de las seis que se resaltan, el incremento de poder del cuerpo permanente con tareas ejecutivas así como los controles de identidad o las autorizaciones y denegaciones de entrada en territorio Schengen. La tercera medida novedosa que nos presentan, era la creación de las antenas ${ }^{6}$ que en el futuro apoyarán las actividades operativas de FRONTEX tanto en los Estados miembros como en terceros países no pertenecientes a la UE. En cuarto lugar, se vislumbra una fuerte apuesta por la externalización de la frontera exterior europea, mediante la ampliación del mandato de la Agencia, que regula la cooperación con terceros países no pertenecientes a la UE y que no tienen que ser necesariamente Estados vecinos. La quinta medida novedosa de la Comisión en su nota de prensa fue el anuncio del aumento del apoyo en los procedimientos de retorno, esta se realiza mediante la recopilación de la información necesaria para emitir decisiones de devolución e identificación de nacionales no pertenecientes a la UE que no puedan permanecer en territorio europeo proporcionándoles documentos de viaje, que incluye la financiación de las operaciones de retorno. En último lugar, se muestra como novedad la compra de su propio equipamiento como buques o aviones.

Para algunos miembros de la Comisión, este nuevo reglamento es una transformación que desarrolla por completo la $\mathrm{GEFC}^{7}$, afirmación de la que, tal como analizamos más tarde, no es del todo acertada ya que la Agencia continúa limitada por las decisiones unilaterales de los Estados miembros y la nueva normativa afianza aún más su labor.

Durante la tramitación legislativa del reglamento, se puede observar cómo los legisladores europeos, tratan de justificar la nece-

5 Puede consultarse el texto en la página oficial de la Comisión europea. https:// ec.europa.eu/commission/presscorner/detail/en/statement_19_6237. Consultado el 19 de noviembre de 2019.

6 Oficinas para gestionar y coordinar los recursos humanos y técnicos y prestar apoyo operativo en las actividades de la Agencia en zonas de operaciones. Figura recogida en el art. 60 del Reglamento (UE) 2019/1896.

7 Puede consultarse el texto en https://ec.europa.eu/commission/presscorner/ detail/en/statement_19_6237. Consultado el 19 de noviembre de 2019. El Comisario de Migración, Asuntos de Interior y Ciudadanía Dimitris Avramopoulos manifestó: "Today the European Union has achieved an ambitious task of transforming the EU boder agency, Frontex, into a fully-fledged European Border an Coast Guard." 
sidad de este nuevo texto normativo en un período de tiempo tan corto, y lo motivan reconociendo el fracaso de la versión 1.0 de la GEFC. Se fundamenta el fracaso en la falta de solidaridad de los Estados miembros que no han contribuido ni con personal ni con equipos suficientes para que la Agencia sea eficaz en las operaciones conjuntas. Además, en estos dos años desde la creación de la GEFC se han constatado incoherencias en la aplicación de la estrategia de gestión europea integrada de las fronteras (en adelante GEIF) por parte de los Estados miembros ${ }^{8}$. En esta misma línea se reconoce la soberanía plena de los Estados miembros y por ello la Comisión propone la creación de un cuerpo permanente propio con competencias ejecutivas, que evite así la dependencia de los Estados miembros. De este modo, trata de constituir su propio brazo operativo, por lo que absorbe el reglamento EUROSUR y amplía su funcionamiento con la esperanza de no tener que depender de ningún Estado. Este intento de empoderamiento ejecutivo de FRONTEX, se ha visto cercenado por las modificaciones introducidas en el texto, y que no han hecho sino agregar numerosas adiciones en el articulado en las que se alude a la necesaria autorización del Estado miembro de acogida.

\section{EL FINGIDO AUMENTO DE LA CAPACIDAD DE LA GEFC.}

\section{II.A. Capacidad operativa.}

Si sólo pudiéramos describir con una única referencia la versión 2.0 de la GEFC, esa no sería otra que 10.000 que es el número de efectivos con el que contará la Agencia a través de un cuerpo permanente $^{9}$ en el año 2027. Esta cifra ha sido muy reiterada en el último año ${ }^{10}$ como seña de identidad del nuevo reglamento e infalible solución a todas las flaquezas que ha mostrado FRONTEX desde la crisis migratoria del año 2015.

8 Resolución del Parlamento Europeo de 30 de mayo de 2018, sobre el informe anual de funcionamiento del espacio Schengen, que indicaba la necesidad de introducir la estrategia de gestión europea integrada de forma rápida y completa.

9 En el nuevo reglamento se encuentran 108 referencias al cuerpo permanente que denotan su importancia.

10 Como decía en nota de prensa de la Comisión el 8 de noviembre de 2019 Frans Timmermans: "De menos de 300 guardias fronterizos en el terreno en 2014, la Guardia Europea de Fronteras y Costas está ahora desplegando alrededor de 1.300 oficiales y pronto tendrá un cuerpo permanente de 10.000 miembros disponibles para despliegue. Este es un logro colectivo, que no hubiera sido posible sin un fuerte apoyo político para un enfoque común". 
El cuerpo permanente viene recogido en el capítulo segundo, sección novena, que hace referencia a las capacidades y trata de la expresión normativa de la tan perseguida GEFC. Se estructura su composición en cuatro categorías de personal operativo; (personal estatutario, personal operativo en comisión de servicio de larga duración, personal operativo para despliegues de corta duración y personal de reserva de reacción rápida) ${ }^{11}$. Todo este personal ya está siendo reclutado en los Estados miembros como se realizaba con el derogado reglamento de la GEFC, pero introducen una gran novedad, con la intención de evitar los problemas encontrados hasta ahora en la Agencia para la cesión de personal por parte de los Estados miembros a FRONTEX, se crea una categoría (1) de personal estatutario propio, que suprime esa dependencia y desarrolla autonomía en el reclutamiento de personal. Como se puede observar en el Anexo I del reglamento, el personal estatutario se irá incrementando de forma progresiva hasta llegar a los 3.000 efectivos en el año 2027, lo que viene a duplicar los cerca de 1.300 guardias de fronteras actuales. Las tres categorías restantes de personal (2, 3 y 4), al finalizar el período formativo del cuerpo permanente conformarán el resto del montante de 7.000 efectivos que no son propios de la Agencia y que deben ser designados por los Estados miembros hasta completar la cantidad final de 10.000 efectivos. Con estos datos podemos constatar que el cuerpo permanente nace y arrastra los mismos problemas que presentaba FRONTEX con el anterior reglamento. De los 10.000 efectivos no podrá disponer de 7.000 contra la voluntad de los Estados miembros debido a que tienen que ser designados por estos. Se duplica su capacidad operativa actual, pero ante una situación migratoria grave dependerá de la solidaridad europea que en estos últimos años ha escaseado.

Este nuevo cuerpo permanente es lo más cercano que ha estado la Unión Europea de la creación de un cuerpo policial de fronteras europeo cuando, en el año 1998, surgió una propuesta al respecto ${ }^{12}$. Aunque parezca que el objetivo está cercano, la realidad es muy diferente, pese a que algunos mensajes institucionales quieran ser más optimistas al respecto. En primer lugar, la tentativa de uniformar al cuerpo permanente de FRONTEX para así subrayar el carácter supranacional de este personal y diferenciarlo del personal estatal. Tibio intento, pues la Agencia solo ha resuelto uniformar al personal

11 Considerando (58) Reglamento UE 2019/1896 sobre la Guardia Europea de Fronteras y Costas.

12 Resolución de 3 de abril de 1998 sobre las repercusiones de la ampliación de la Unión Europea en lo que respecta a la cooperación en los ámbitos de la justicia y de los asuntos de interior, DOCE n C138 de 04.05.1998, A4-0107/98. 
estatutario (3.000 efectivos) abandonando al resto del cuerpo permanente (7.000 efectivos) con su uniforme nacional propio con el añadido de un brazalete azul con la bandera de la Unión europea y el escudo de FRONTEX ${ }^{13}$ tal y como se hacía hasta ahora. En el considerando (71) del nuevo reglamento, encontramos otro intento de reforzar ese cuerpo de personal permanente cuando se afirma que está acompañado por la facultad de adquisición, mantenimiento y uso de medios aéreos, marítimos y terrestres propios de la Agencia, llega a situarlos como "espina dorsal" de los despliegues operativos. Ésta línea de actuación ya iniciada se puede comprobar con la adquisición reciente de vehículos que ya han sido utilizados en operaciones en Albania, Grecia y Bulgaria ${ }^{14}$. No obstante, la Agencia aún está muy lejos de poseer, en la práctica, una plena autonomía de medios materiales.

El cuerpo permanente afianza y mejora el funcionamiento y procedimiento de sus miembros desde el punto de vista puramente operativo y determina con precisión vacíos legales no recogidos con anterioridad. Por un lado, se amplía el ámbito territorial con la posibilidad de despliegue básico del cuerpo permanente ejecutado mediante equipos que se podrán situar en territorios de Estados miembros o de terceros países ${ }^{15}$, Aunque en el derogado reglamento permitía que los equipos se desplegaran en terceros países, la realidad era que no se ejercía de forma correcta esta función, al dejar vacíos legales que eran aprovechados por FRONTEX para dejar a sus efectivos en territorio extranjero en un limbo legal de responsabilidad. El nuevo reglamento reordena estos procedimientos y concreta su ejecución y control. Esta cuestión será desarrollada en profundidad en el apartado IV. Otro cambio es la modificación de los equipos que a partir de ahora pasarán a estar formados por guardias de fronteras, escoltas para retornos, especialistas en retorno y otros necesarios, ${ }^{16}$ estructuran sus funciones organizadas en tres tipos de equipos (gestión de fronteras, apoyo a la gestión de la migración y retorno) $)^{17}$, aquí sí es perceptible un ajuste basado en la experiencia operativa de los últimos años, se simplifica el modelo que

13 Artículo 82.6 del reglamento (UE) 2019/1896 sobre la Guardia Europea de Fronteras y Costas.

14 Puede consultarse en Memoria FRONTEX 2019. Consultada el 27 de marzo de 2020. https://frontex.europa.eu/assets/Publications/General/In_Brief_2019/frontex_inbrief_ES.pdf.

${ }_{15}$ Considerando (66) y art. 73.3 del reglamento (UE) 2019/1896 sobre la Guardia Europea de Fronteras y Costas.

${ }_{16}$ Artículo 2 apartado 16) definición de personal operativo reglamento (UE) 2019/1896 sobre la Guardia Europea de Fronteras y Costas.

17 Artículo 2 apartado 17) definición de miembro de los equipos en reglamento (UE) 2019/1896 sobre la Guardia Europea de Fronteras y Costas. 
se venía utilizando con anterioridad y que distribuía los equipos de actuación en las tres funciones principales que desarrolla la Agencia. Por último, en aras de clarificar la operativa del nuevo cuerpo y lograr solucionar los problemas encontrados hasta el momento con experiencias en las diferentes operaciones realizadas, se incluye como novedad un Anexo V que versa sobre "normas relativas al uso de la fuerza, incluida la formación, el suministro, el control y el uso de armas de servicio y de equipamiento no letal, aplicables al personal estatutario desplegado como miembro de los equipos". Un nuevo apoyo reglamentario en la utilización de la fuerza por el nuevo cuerpo en operaciones en suelo de Estados miembros de acogida, pero siempre sin olvidar que el uso de la misma por los miembros de FRONTEX, sólo podrá realizarse en presencia de los guardias de fronteras del Estado miembro y con la previa autorización de las autoridades nacionales de acogida, salvo que fueran exonerados expresamente de tal requisito presencial ${ }^{18}$. Tiempo atrás, nada se preveía en el reglamento con respecto a estas situaciones.

\section{II.B. Capacidad financiera.}

En el único aspecto que no deja lugar a dudas en cuanto al fortalecimiento operativo de FRONTEX y la creación del cuerpo permanente, es el financiero. Del presupuesto de la UE para el año 2020 se destinarán a seguridad y gestión de la inmigración 2.360 millones de euros ${ }^{19}$. De estas funciones se ocupan diversas agencias europeas como EASO, EUROPOL o FRONTEX, entre otras. Es importante resaltar el esfuerzo económico de la Unión Europea que asciende a 460 millones de euros que se invierten en la Agencia y que constituyen un $19 \%$ del total de la partida según las previsiones de la programación de FRONTEX para el período 2020-2022 (Tabla 2). Desde su creación en el año 2004 el presupuesto de la Agencia ha crecido de forma moderada hasta el año 2015 en el que lo hizo de manera exponencial, debemos situar todo ello coincidiendo con la grave crisis migratoria y la reconversión de FRONTEX en la GEFC, esto genera un punto de inflexión presupuestario en la Agencia que en los últimos años ha hecho que no pare de aumentar. Este último aspecto se refleja sustancialmente en el cambio que se produce en la programación previa

18 Punto 3 del Anexo V del Reglamento (UE) 2019/1896 sobre la Guardia Europea de Fronteras y Costas.

19 Puede consultarse el texto en: https://www.expansion.com/economia/2019/11/ 19/5dd32e30e5fdea8c048b45c6.html. Consultado el 8 de abril de 2020. 
(Tabla 1) ya que las propias previsiones para el ejercicio 2020 se incrementarán en 115 millones de euros en sólo un año (Tabla 2).

Tabla 1 Programación FRONTEX 2019-202120.

\begin{tabular}{|c|c|c|c|c|c|}
\hline & & 2017 & 2018 & 2019 & 2020 \\
\hline & & $\operatorname{COM}(2015) 671$ & $\operatorname{com}(2015) 671$ & $\operatorname{COM}(2015) 671$ & $\operatorname{com}(2015) 671$ \\
\hline \multirow[t]{2}{*}{ Subvención de la CE } & Compromisos & 281267 & 298286 & 310289 & 322227 \\
\hline & Pagos & 281267 & 298286 & 310280 & 322227 \\
\hline \multirow{2}{*}{$\begin{array}{l}\text { Contribución de los PAS } \\
\text { (Importe indicativo) }\end{array}$} & Compromisos & 20662 & 21912 & 22794 & 23672 \\
\hline & Pagos & 20662 & 21912 & 22794 & 23,672 \\
\hline \multirow{2}{*}{$\begin{array}{c}\text { TOTAL } \\
\text { (Importo indicativo) }\end{array}$} & Compromisos & 301929 & 320198 & 333083 & 345899 \\
\hline & Pagos & 301929 & 320198 & 333083 & 345899 \\
\hline
\end{tabular}

Tabla 2 Programación FRONTEX 2019-202121.

\begin{tabular}{|l|l|c|c|c|}
\cline { 3 - 5 } \multicolumn{2}{c|}{} & 2020 & 2021 & 2022 \\
\hline \multirow{2}{*}{ EUR million (to three decimal places) } & $10919 / 19$ ADD & COM(2018)631 & COM(2018)631 \\
\hline \multirow{2}{*}{ EC contribution } & Commitments & 428,160 & 846,906 & $1,043,043$ \\
\cline { 2 - 5 } & Payments & 428,160 & 846,906 & $1,043,043$ \\
\hline \multirow{2}{*}{$\begin{array}{l}\text { SAC contribution } \\
\text { (indicative amount) }\end{array}$} & Commitments & 31,795 & 54,058 & 66,577 \\
\cline { 2 - 5 } & Payments & 31,795 & 54,058 & 66,577 \\
\hline \multirow{2}{*}{$\begin{array}{l}\text { TOTAL (indicative } \\
\text { amount) }\end{array}$} & Commitments & 459,955 & 900,964 & $1,109,620$ \\
\cline { 2 - 5 } & Payments & 459,955 & 900,964 & $1,109,620$ \\
\hline
\end{tabular}

La escalada presupuestaria no sólo se mantendrá los próximos años, sino que en el ejercicio 2021 se estima que se duplicará hasta los 900 millones de euros, y llegará a su máximo histórico en el año 2022 con 1.109 millones de euros.

Esta potentísima inversión económica no sustenta una ampliación del mandato de FRONTEX que le reporte asumir nuevas competencias, solo es la base de un crecimiento muy importante en materia de recursos humanos, y de forma secundaria, en recursos materiales que busca un reforzamiento securitario sin asegurar la eficiencia y además sin que se avance en la integración europea de fronteras exteriores. Un ejemplo de ello tuvo lugar en una operación conjunta en uno de los puntos críticos de Italia donde se estaban

20 Puede consultarse el texto en: https://frontex.europa.eu/assets/Key_Documents/Programming_Document/2019/Programming_Document_2019_2021_ES.pdf . Consultado el 8 de abril de 2020.

21 Puede consultarse el texto en: https://frontex.europa.eu/assets/Key_Documents/MB_Decision/2019/MB_Decision_31_2019_adopting_Programming_Document_2020_2022.pdf. Consultado el 8 de abril de 2020. 
produciendo llegadas por mar. FRONTEX desplegó la presencia de su personal muy por encima de las necesidades en ese momento, en el que no se reportaban casi llegadas y el punto crítico estaba cerca de estar vacío ${ }^{22}$.

Todos los datos y cifras expuestos en este primer apartado deberán de tomarse con cierta cautela, ya que son previsiones oficiales a medio o largo plazo proyectadas en un período que abarca hasta el año 2027 y es frecuente que las políticas europeas en materia de seguridad o migración se vean afectadas en el corto plazo por decisiones de urgencia, un ejemplo de ello se produce en materia presupuestaria cuando se revisan anualmente las previsiones para incrementos presupuestarios que alcanzan los 115 millones de euros y van mucho más allá de los meros ajustes. El propio reglamento ordena a la Comisión que se revise la composición del cuerpo permanente, así como las contribuciones que ha realizado cada Estado miembro al mismo y marca como fecha límite para su presentación antes del 31 de diciembre de $2023^{23}$. En esa misma línea, la Comisión antes del 5 de diciembre de 2023, habrá de proceder a la evaluación de su funcionamiento ${ }^{24}$ y no resultaría extraño que surgieran modificaciones importantes en la planificación prevista.

\section{EL TECHO DE LA COMPETENCIA COMPARTIDA.}

La imagen que trasladan las autoridades europeas respecto de las nuevas competencias de la Agencia, responde a un exagerado optimismo cercano a la euforia, que no se corresponde con la realidad. Así, podemos leer declaraciones del director ejecutivo de FRONTEX para el diario "El Mundo", en las que considera "histórico" el lanzamiento del cuerpo permanente. "Colegas europeos llegando con un uniforme europeo...jEso es Historia!"25. Más lejos llega el comisario griego Margaritis Schinas en un encuentro con periodistas españoles en diciembre de 2019 en el que proclama "Con esta Guardia Europea de Fronteras y Costas se federaliza el control de fronteras externas de

22 Informe especial no 24/2019 del Tribunal de Cuentas Europeo pág.17 ISBN 978-92-847-3966-0

23 Artículo 59 del reglamento (UE) 2019/1896 sobre la Guardia Europea de Fronteras y Costas.

${ }^{24}$ Artículo. 121.1 apartado f) reglamento (UE) 2019/1896 sobre la Guardia Europea de Fronteras y Costas.

25 Puede consultarse el texto en: https://www.elmundo.es/internacional/2019/12/ 06/5de9517621efa0275a8b4594.html. Consultado el 9 de abril de 2020. 
la $U E^{\prime 26}$. Este tipo de declaraciones se alejan de la realidad jurídica, que muestra un sistema de competencias compartidas ${ }^{27}$ con una sólida base intergubernamental y que está muy lejos de la estructura federal que se pretende trasmitir.

\section{III.A. El artículo 4.2 del TFUE y su encaje en la versión 2.0 de la GEFC.}

La federalización del control de fronteras choca de facto, con el artículo 4.2 del Tratado de la Unión Europea (en adelante TUE) ${ }^{28}$, que limita a la Unión y exige respeto para los Estados miembros en lo referente a las funciones esenciales que garanticen su integridad territorial como competencia exclusiva estatal. El derogado reglamento de la GEFC respetaba este precepto, ${ }^{29}$ así como el artículo 4.2 del TFUE, en el que se atribuye como competencia compartida el Espacio de Libertad, Seguridad y Justicia (ELSJ), encuadrándose dentro de este espacio también el artículo 77 del TFUE que versa sobre la gestión integrada de fronteras exteriores. En concordancia con las normas anteriores, el nuevo reglamento UE 2019/1896 en su artículo 7, mantiene la responsabilidad compartida en tareas de control fronterizo por parte de la Agencia y de las autoridades nacionales responsables. Queda claro en la versión 2.0 de la GEFC, que continua esa misma línea legislativa sin restar competencias a los actores estatales ${ }^{30}$ y tampoco aumenta las de la Agencia. Nos encontramos en una nueva fase de la integración de la gestión de las fronteras europeas que no avanza y que vuelven a basarse en un único

26 Puede consultarse el texto en: https://www.elindependiente.com/politica/europa/2020/01/01/se-buscan-guardacostas-made-in-ue-frontex-se-reinventa-y-ampliasus-miras/. Consultado el 9 de abril de 2020. En ese mismo encuentro también manifiesta: "Serán el primer cuerpo comunitario con su propio equipamiento, barcos, armas... Llevarán uniformes comunitarios".

27 DEL VALLE GÁLVEZ, A., "Las fronteras de la Unión. El modelo europeo de fronteras", Revista de Derecho Comunitario Europeo, ${ }^{\circ} 12$ (mayo-agosto 2002), pág. 320.

${ }_{28}$ MANGAS MARTIN, A., “Territorio, integridad territorial y fronteras del Estado en la Unión Europea”, Revista Jurídica de la Universidad de León, n 2, 2015, pág. 234.

${ }_{29}$ PEERS, S., "The Reform of Frontex: Saving Schengen at Refugees' Expens?", en EU Law Analysis, Puede consultarse el texto en: www.eulawanalysis.blogspot. it,2015,p.3. Consultada el 9 de abril de 2020.

30 FERNÁNDEZ ROZAS, J.C., "Control de fronteras, asilo e inmigración en la Unión Europea: un conflicto competencial no resuelto", Memorial para la Reforma del Estado. Estudios en homenaje al profesor Santiago Muñoz Machado, t. I, Madrid, Centro de Estudios Constitucionales, 2016, pág. 369. 
incremento de capacidad de recursos humanos y materiales ${ }^{31}$, como ya ocurriera en el año 2016.

Cuando revisamos el objeto de la GEFC que se implanta en el remodelado artículo 1 del nuevo reglamento, apreciamos un cambio de adverbio a la hora de garantizar la gestión integrada de las fronteras exteriores, se sustituye el hasta ahora utilizado "eficazmente" por el nuevo "eficientemente". Este simple cambio muestra una paradoja entre los dos textos. Mientras en el primero se utilizaba "eficazmente", la recién creada en ese momento GEFC no disponía de los suficientes medios ni humanos ni materiales, pero confiaba en que los Estados miembros se los proporcionarían y sin embargo, se sentía con el poder suficiente para controlar la situación y dirigir la securitización de Europa. Por eso pretendía la "eficacia". Actualmente, en el segundo reglamento los elementos han cambiado, por un lado trata de proveerse de recursos humanos y materiales propios para deshacerse de la dependencia de los Estados miembros, pero sin embargo pese a conseguir un potencial humano y material muy importante es consciente que no tiene el poder de dirigir la situación debido a su dependencia de los Estados miembros que siempre tendrán la última palabra antes de que pueda intervenir en el terreno. Por esa razón utiliza la "eficiencia", porque van a incrementarse los medios, pero van a tener que coordinarse y ser utilizados en su justa medida, ya que al existir dos actores implicados que van a intentar actuar, pueden aparecer disfunciones en las que se vislumbra que el Estado miembro impondrá su soberanía.

\section{III.B. Idénticas tareas en las versiones 1.0 y 2.0 de la GEFC.}

Para analizar si existe un incremento en las tareas ejecutivas que presenta el reglamento en vigor de la GEFC y de su predecesor ya derogado $^{32}$, el procedimiento más simple y directo es comparar los artículos 10 y 8 de ambos. Una primera aproximación a ambos artículos nos podría llevar a pensar que en efecto se ha ampliado el mandato de FRONTEX. Mientras que el antiguo artículo 8 enumera en su punto primero hasta 22 tareas, el nuevo artículo 10 presenta 33 tareas de la Agencia Europea de la Guardia de Fronteras y Cos-

31 DE CASTRO SANCHEZ, C., "Gestión integrada de fronteras, FRONTEX y derechos fundamentales. ¿Un nuevo escenario tras el Reglamento (UE) 2016/1624?”. Tirant lo Blanch, Valencia 2018. pág 391.

32 FERNÁNDEZ ROJO, "Los poderes ejecutivos de la Guardia Europea de Frontera y Costas: del Reglamento 2016/1624 al Reglamento 2019/1896, en Revista catalana de dret públic, nº 60, pp. 181-185. 
tas. Las matemáticas no fallan, se trata de once tareas más que en el antiguo mandato por lo que estamos ante un incremento evidente. Sin embargo, al analizar ambos artículos en profundidad observamos que no existe tal ampliación de competencias. De las 33 tareas de la versión 2.0 de la GEFC sólo once ${ }^{33}$ son presuntamente novedosas si atendemos al Anexo VI de la tabla de correspondencias del Reglamento UE 2019/1896. Esa presunción de novedad se desvirtúa en el momento en el que profundizamos en su contenido, muchas de las denominadas nuevas tareas resultan ser concreciones técnicas o repartos operativos necesarios después de la experiencia obtenida estos últimos años en el trabajo de campo a todas ellas las podemos clasificar en tres bloques.

\section{III.B.1. Tareas retocadas}

En un primer bloque, situamos tareas que puntualizan o concretan la operativa de la Agencia. Por ejemplo, es el caso del art. 10.1 apartado b), (inexistente con anterioridad según la tabla de correspondencias), que versa sobre la supervisión de necesidades operativas de los Estados miembros en la aplicación de retornos. Este apartado tiene mucha correlación con el apartado o) "crear un contingente de supervisores de retorno forzoso" que si se encuentra indexado con el antiguo artículo 8.1 apartado n) que trata de la "creación de contingentes de supervisores de retorno forzoso, escoltas y especialistas en retorno". Lo que hacen los legisladores en estos apartados que acabamos de señalar, es ampliar el rango de tarea a un nivel superior de supervisión por la Agencia, en cuanto a los sujetos afectados. Se supervisan las necesidades de los Estados miembros en los retornos en general (no solo en los forzosos) y en estos últimos, mantiene su propio contingente de supervisores de retorno forzoso. Es evidente que no se produce un incremento de tarea ejecutiva puesto que el Estado miembro mantiene el poder de decisión.

\section{III.B.2. Tareas existentes puestas en valor.}

En el segundo bloque, tenemos tareas que ya existían en la normativa sectorial y que se han elevado al artículo referido a las tareas para procurarles más notoriedad y constancia de la importancia que les otorga el legislador. Además, son complementadas con principios

33 Artículo 10.1 apartados (b, e, l, r, s, u, y, ad, ae, af, ag) del reglamento (UE)2019/1896. 
transversales que buscan la mejora del funcionamiento interno de la Agencia. Encontramos varios apartados que poca o nula repercusión tienen frente a terceros. Un ejemplo que trata de elevar la notoriedad de la tarea, es el apartado e) del artículo 10.1 que asigna la supervisión del respeto de los derechos fundamentales en las actividades que se puedan producir en las fronteras exteriores u operaciones que intervenga FRONTEX. En el anterior reglamento, su artículo 34 establecía unas normas generales sobre la protección de los derechos fundamentales y la estrategia sobre los mismos, que ha tenido continuidad con el artículo 80 del nuevo texto, la novedad consiste en dar relevancia a la garantía de los derechos fundamentales, que sitúan el nivel de supervisión en el artículo 10 y recogen todas las tareas de la GEFC. A mi juicio, esta modificación no es apenas relevante desde el punto de vista ejecutivo. El fortalecimiento de las garantías y de la visibilidad de los derechos humanos sigue la tendencia general de otras agencias e instituciones de la UE, pero en la práctica, la Agencia ya venía desarrollando sus operaciones respetando el marco establecido por la Carta de derechos fundamentales. En todo caso, estas cuestiones necesitarían una reflexión en profundidad (que excede la finalidad y objeto de este artículo), para analizar por ejemplo, el grado de la responsabilidad de la Agencia ante actuaciones de guardias de fronteras y costas bajo el mandato de operaciones conjuntas de FRONTEX ${ }^{34}$.

Los principios transversales complementados que se han mencionado en las líneas previas, se presentan en varios apartados del artículo 10.1 del nuevo reglamento que serían recomendables para cualquier organización (apartados l, r, s, y, ad) y que tratan de principios como control de calidad, cooperación, normas técnicas de comunicación o transparencia. El apartado l), introduce un mecanismo interno de control de calidad para gestionar las capacidades técnicas y humanas del cuerpo permanente, muy necesario pero irrelevante en cuanto al incremento de tareas ejecutivas. Los apartados r) y s) por su parte se encargan de asegurar una cooperación institucional con otras agencias europeas del Espacio de Libertad Seguridad y Justicia, en concreto con EASO y FRA, con las que ya se venía cooperando en el pasado y deja plasmado en el nuevo reglamento, una práctica que ya se ejercía en ámbitos como por ejemplo, los de-

34 Ver entre otros MUNGIANU, R. "Frontex y no devolución: La responsabilidad internacional de la UE", Cambridge Studies in European Law and Policy, p. I. Cambridge: Cambridge University Press. (2016). BROOKS, TEQUILA J., "Can Frontex be held liable for human rights violations? Potential application of recent European case law to the activities of an intergovernmental agency" (2012). Puede consultarse el texto en: https://papers.ssrn.com/sol3/papers.cfm?abstract_id=2088249. 
rechos fundamentales a través del foro consultivo de FRONTEX. El apartado y) por su parte, ordena el desarrollo de normas técnicas para el intercambio de información dentro y fuera de la propia Agencia y es también claro ejemplo de la falta de relevancia de este incremento de tareas con estos apartados, que por otro lado si cimentan un mejor funcionamiento operativo de la Agencia.

\section{III.B.3. Tareas auxiliares.}

Por último, el tercer bloque con el que he clasificado las tareas (presuntamente) novedosas que presenta el artículo 10, se compone de dos instrumentos auxiliares de gestión de las fronteras que a simple vista parecen un importantísimo incremento en las tareas de la Agencia, pero que serán de gran ayuda para las labores de FRONTEX, sin que esto conlleve más expansión ejecutiva.

\section{III.B.3.a) Sistema de Documentos Auténticos y Falsos en Red (FADO).}

En el artículo 10.1, apartado ae) encontramos una de las tareas que contiene el primero de estos instrumentos y en el que además se puede apreciar la falta de poder ejecutivo de la GEFC, es el Sistema de Documentos Auténticos y Falsos en Red, en lo sucesivo (FADO) ${ }^{35}$, creado mediante acción común 98/700/JAI del Consejo el 3 de diciembre de 1998, por la que se instauraba un Sistema europeo de archivo de imágenes. Su objetivo era intercambiar información sobre documentos auténticos y falsos entre los Estados miembros. Esta acción común ha quedado derogada por el nuevo reglamento UE 2020/493 de fecha 30 de marzo $^{36}$, en el que se encarga a FRONTEX el control y explotación del sistema FADO por sus conocimientos especializados en esta materia ${ }^{37}$. En previsión y consonancia con esta línea legislativa, encontramos la versión 2.0 de la GEFC, que en su capítulo tercero con un único artículo 79, en el que se mandata a la Agencia a asumir y explotar el sistema FADO conforme a la ya derogada acción común 98/700/JAI. En las conclusiones del Consejo de 27 de marzo de 2017, se declaraba la obsolescencia de la gestión del

35 Reglamento (UE) 2020/493 del Parlamento europeo y del Consejo de 30 de marzo de 2020 relativo al sistema FADO. Publicado en el DOUE el 6 de abril de 2020, con entrada en vigor el 26 de abril de 2020.

36 Artículo 9 del reglamento (UE) 2019/1896 de la Guardia Europea de Fronteras y Costas.

37 Considerando (13) Reglamento 2020/493 de 30 de marzo. 
sistema y se proponía que la Agencia se hiciera cargo de la administración y gestión tanto operativa como técnica de FADO hasta que se sustituyese la acción común del año $1998^{38}$.

Aun pudiendo ser el fraude documental una labor incardinable en el ámbito competencial de otras agencias europeas de cooperación policial, como es el caso de EUROPOL, se ha decidido que la más preparada para la lucha contra la falsedad documental es FRONTEX por su experiencia adquirida en los últimos años y por su detección frecuente en las fronteras exteriores. Lo que en un principio puede parecer un incremento de competencias ejecutivas, al final resulta ser un artificio más del tan ansiado poder ejecutivo que pretende ostentar la Agencia.

El sistema FADO, no deja de ser un instrumento auxiliar para las funciones operativas del futuro cuerpo permanente de la GEFC, al constituir una herramienta informática de consulta sobre documentos de viaje, de residencia, permisos de conducción y otros expedidos por instituciones de la UE, Estados miembros o terceros estados que quieran participar en el sistema. FADO pese a su indispensable utilidad operativa, no deja de ser un sistema secundario en las importantes misiones que FRONTEX desarrolla, como por ejemplo la protección de fronteras exteriores. En este caso, la Agencia ha sido instrumentalizada para que se haga cargo de un sistema en el que va a tener muy poco control debido a que otros actores como la Comisión o los Estados miembros van a determinar el devenir del nuevo sistema FADO. La Comisión, en el nuevo reglamento UE 2020/493 que desarrolla el art. 79 del último reglamento de la GEFC asume actos ejecutivos como establecer la arquitectura técnica, las especificaciones técnicas de introducción y almacenaje de datos, procedimientos de control y verificación de la información e incluso fijará la fecha de aplicación efectiva del nuevo sistema por la Agencia ${ }^{39}$. También, adoptará las medidas oportunas que permitan el acceso al sistema y los procedimientos necesarios para ello como por ejemplo; obligar a la firma de acuerdos entre la Agencia y un tercero o entidad privada ${ }^{40}$. A simple vista, el papel de FRONTEX es casi de mero introductor de datos en el sistema y así se constata cuando entra en juego el segundo actor, que no son otros que los todopoderosos Estados miembros que vacían de poder de acción a la Agencia. En primer lugar, tendrán que aprobar la transmisión de la información

38 Considerando (96) Reglamento 2019/1896 de la Guardia Europea de Fronteras y Costas.

39 Artículo 6 del reglamento 2020/493 de 30 de marzo relativo a FADO

40 Artículo 4.5 del reglamento 2020/493 de 30 de marzo relativo a FADO. 
que contiene en la actualidad el sistema FADO y que sin su autorización no podrá formar parte de la nueva gestión que inicia la Agen$\mathrm{cia}^{41}$. Cada uno de los Estado miembros además, debe determinar que autoridades nacionales son las que accederán al sistema y con que nivel de acceso, siendo totalmente autónomos en esta designación ${ }^{42}$. Para evitar cualquier tipo de conflicto futuro entre la Agencia y los Estados miembros, el considerando (15) del reglamento UE 2020/493, blinda la competencia de reconocimientos de pasaportes, documentos de viajes, visados y otros... que recae sobre los Estados miembros sin ningún género de dudas. Por último, encontramos como obligación de los Estados miembros, la transmisión sin demora de la información sobre los documentos que versa este reglamento ${ }^{43}$, pero sin establecer ningún instrumento que los pueda forzar ante su negativa a facilitar información, y la Agencia irremediablemente deberá recurrir en ese caso a los procesos generales de incumplimiento de los reglamentos europeos.

Es evidente que el nuevo sistema FADO incluido en la versión 2.0 del nuevo reglamento de la GEFC, no atribuye ninguna potestad ejecutiva novedosa a FRONTEX que no vaya más allá de mantener una base de datos actualizada y lista para su uso operativo por los diferentes integrantes del sistema, como puedan ser cuerpos policiales, guardias de fronteras y otras autoridades nacionales.

\section{III.B.3.b). Sistema Europeo de Información y Autorización de Viajes (SEIAV).}

El otro instrumento nuevo que presenta el artículo 10.1 apartado af), es el Sistema Europeo de Información y autorización de Viajes (en adelante SEIAV y también conocido por sus siglas en inglés como ETIAS). Será un futuro sistema de información automatizado gestionado por la agencia europea eu-LISA, ${ }^{44}$ que administra sistemas de información a gran escala en las áreas del Espacio de Seguridad, Libertad y Justicia. SEIAV controlará la entrada y salida de nacionales de terceros países exentos de la obligación de obtener visado para cruzar las fronteras exteriores y deambular por territorio Schengen.

41 Artículo 9.3 del reglamento2020/493 de 30 de marzo relativo a FADO.

42 Artículo 4.6 del reglamento 2020/493 de 30 de marzo relativo a FADO.

43 Artículo 2.3 del reglamento 2020/493 de 30 de marzo relativo a FADO.

44 Agencia Europea para la Gestión Operativa de Sistemas Informáticos de Gran Magnitud en el Espacio de Libertad, Seguridad y Justicia, cuya función es la gestión de sistemas informáticos de gran magnitud de la UE, operativa desde 1 de diciembre de 2012. 
En la actualidad, nacionales de 60 países están afectados por la exención de la necesidad de visado, pero en un futuro próximo (entrará en vigor a finales del año 2022) ${ }^{45}$ estarán obligados a obtener el permiso de viaje SEIAV para entrar en territorio Schengen.

Se tratará de un instrumento importante dentro de la GEIF, que permitirá el control de movimientos de un segmento de pasajeros con unas características administrativas concretas como la exención de visado. La Unión decidió que FRONTEX tuviera un papel preponderante en este nuevo sistema y desde el 9 de octubre de 2018, introdujo modificaciones legislativas en el reglamento de la GEFC vigente en ese momento. La modificación consistió en introducir un apartado y un artículo nuevo. El apartado que se añadió fue en el artículo 8.1 del Reglamento UE 2016/1624 dedicado a las tareas de la agencia, un nuevo apartado q bis) en el que se mandaba a la GEFC cumplir las funciones y obligaciones del reglamento UE 2018/1240 del SEIAV. Este mandato se complementó con un nuevo artículo 33 bis) en el que se plasmaba la unidad central SEIAV y se encargaba a la GEFC su creación y funcionamiento.

Por otro lado, la normativa del SEIAV crea varios órganos propios que sitúa dentro de la agencia GEFC. El más importante y que gestionará el sistema, es una unidad central que deberá funcionar las 24 horas del día los 7 días de la semana ${ }^{46}$. Este mandato enlaza con el nuevo reglamento GEFC en su versión 2.0 a través del artículo 67 que garantiza la creación y funcionamiento de la unidad central, (según se contempla en el artículo 7 del reglamento SEIAV). El segundo órgano que encontramos en la GEFC es un Consejo de Detección con funciones consultivas ${ }^{47}$. Por último, también se crea un Consejo de Orientación sobre Derechos Fundamentales del SEIAV, formado por el agente de derechos fundamentales y por un representante del foro consultivo sobre derechos fundamentales, ambos pertenecientes a la GEFC, además de otros miembros de diversas agencias. Todos los gastos y organización de reuniones correrán a cargo de la $\mathrm{GEFC}^{48}$. Hay que reseñar por último el artículo 75, titulado por los legisladores como "responsabilidades de la Agencia GEFC", en el que descargan sobre ella el establecimiento y funciona-

45 Puede consultarse el texto en: https://www.etiasvisa.com/es. Consultado el 10 de abril de 2020.

46 Artículo 7 del reglamento (UE) 2018/1240 del Parlamento europeo y del Consejo de 12 de septiembre de 2018 por el que se establece SEIAV.

47 Artículo 9 del reglamento (UE) 2018/1240 de 12 de septiembre de 2018 SEIAV.

48 Artículo 10 del reglamento (UE) 2018/1240 de 12 de septiembre de 2018 SEIAV. 
miento de la unidad central SEIAV y la obligación de garantizar la gestión segura de los datos almacenados.

El sistema SEIAV ha iniciado su marcha y conforme al mandato encomendado a FRONTEX, la Agencia ha programado la implementación de sus actuaciones para tener preparado todo el día de su inicio, para ello, ya en el año 2021, tiene previsto la contratación de 250 miembros de personal estatutario propio de GEFC más una rotación de 100 empleados y otros 40 empleados externos ${ }^{49}$.

Para darle la relevancia que se merece al instrumento, lo sitúa en el art. 10.1 apartado af) del nuevo reglamento de la GEFC, que contiene la tarea referente al SEIAV, su inclusión en este lugar sí manifiesta la importancia operativa que proporciona, puesto que no olvidemos que es una herramienta fundamental para los guardias de fronteras y costas, que permitirá decidir con respecto a la admisibilidad de todos los nacionales de terceros países exentos de la obligación de visado para viajar al espacio Schengen y si dichos desplazamientos supondrían un riesgo.

Si comparamos este instrumento con el anterior apartado referido a FADO, el SEIAV es una herramienta mucho más potente, pero en el fondo no debemos de olvidar que su tarea ejecutiva es parcial y limitada a la creación de la unidad central con poder funcional pero no decisorio operativo. Es un gran paso en cuanto a integración de fronteras exteriores, puesto que va a permitir un mayor control y coordinación de los ciudadanos que se muevan por espacio Schengen, pero FRONTEX sólo se dedicará a garantizar el funcionamiento correcto del tratamiento de la base de datos.

Por todo ello, consideramos que se ha instrumentalizado a FRONTEX al ser a día de hoy la alternativa más fácil, puesto que es la agencia más potente de las del Espacio de Libertad, Seguridad y Justicia. Se ha vuelto a repetir el patrón de actuación que se ha realizado con FADO y que no es otro, que concentrar los instrumentos operativos en una agencia que presente personal y presupuesto suficiente para acometer la misión. Las tareas ejecutivas no se incrementan en este apartado con respecto a la versión 1.0 de la GEFC que ya recogían el futuro SEIAV. Es evidente que el mayor peso a la hora de poner el sistema en marcha lo soporta la agencia eu-LISA, mientras que FRONTEX sólo se encargará de una unidad central, que no posee poder decisorio en lo relevante de este instrumento.

49 Puede consultarse el texto en Programación de FRONTEX 2019-2021 pág. 69, https://frontex.europa.eu/assets/Key_Documents/Programming_Document/2019/ Programming_Document_2019_2021_ES.pdf. Consultado el 7 de abril de 2020.

(C) UNED. Revista de Derecho UNED, núm. 27, 2021 
Son los Estados miembros a través de sus unidades nacionales los que expiden, deniegan, anulan o revocan ${ }^{50}$ las autorizaciones de viaje y no FRONTEX en la unidad central de SEIAV. Ni siquiera los miembros del nuevo cuerpo permanente en misión conjunta tendrán esta potestad, siendo los guardias de fronteras y costas nacionales, los que tomen las decisiones, salvo que se haya producido una atribución puntual por parte del Estado miembro de acogida.

Es un incremento de tarea ejecutiva porque la Agencia debe crear una unidad central que es nueva y no ha comenzado a funcionar todavía, pero no deja de ser un instrumento informático auxiliar de las tareas verdaderamente ejecutivas que se realizan en los controles de fronteras por los Estados miembros y en casos puntuales por FRONTEX cuando sea requerido para ello.

\section{III.B.4. Miscelánea recopiladora.}

En último lugar, sin posibilidad de clasificación en ninguno de los tres bloques anteriores, encontramos el apartado ag) del artículo 10.1 del nuevo reglamento de la GEFC, que plasma una especie de cajón de sastre que resume la situación de frustración ejecutiva de la Agencia al recoger la siguiente tarea: "ayudar a los Estados miembros a facilitar a las personas el cruce de las fronteras exteriores". Último intento desesperado de hacerse cargo de cualquier parcela competencial que puedan olvidar por el camino los Estados miembros. Con este apartado se proporciona cobertura legal a una zona gris en la que FRONTEX lleva años navegando muy cómoda y que alberga una miscelánea de tareas ambiguas no recogidas en las normas y que en momentos puntuales puede facilitar su intervención para burlar el control de los Estados miembros y la Comisión.

En sí misma, esta técnica legislativa es un recurso muy utilizado en muchos ordenamientos jurídicos y no tiene porque envolver intereses ocultos, puesto que suele incluirse para proporcionar salida a futuros problemas interpretativos, de aplicación o simplemente para remediar olvidos legislativos. Lo que sí llama la atención en este caso es que el anterior reglamento no lo utilizara y se haya introducido ahora con las controversias jurídicas que ha arrastrado FRONTEX con sus intervenciones.

50 Artículos 39, 40 y 41 del reglamento (UE) 2018/1240 de 12 de septiembre de 2018 SEIAV. 
Como se puede comprobar, la versión 2.0 de la GEFC nos muestra una Agencia sin mayores competencias, ni verdaderos poderes ejecutivos. Por todo ello, es un avance hacia la federalización que, en la práctica real, no refleja los profundos cambios anunciados y en definitiva, se mantiene en el FRONTEX $+{ }^{51}$ propugnado respecto del Reglamento 2016/1624.

\section{LA POTENCIACIÓN EN LA EXTERNALIZACIÓN.}

Europa inició hace años una senda que pretendía remediar los problemas migratorios con soluciones fuera de su territorio y con el establecimiento de una Europa fortaleza. Su construcción se inició en el año 1985 con los acuerdos de Schengen que dividían el espacio en dos, uno interior seguro y otro exterior inseguro. Para defender ese espacio apareció FRONTEX en el año 2004 y se ha ido asentando hasta el día de hoy para asumir un papel importantísimo. Dentro de sus funciones, la Agencia vira el rumbo de forma clara hacia una estrategia de militarización y securitización ${ }^{52}$ de los flujos migratorios en la UE. El último episodio más reciente lo tenemos en las operaciones conjuntas con la OTAN como es "SOPHIA" o "IRINI" que acaban de ser puestas en marcha.

\section{IV.A. Gestión Europea Integrada de Fronteras (GEIF). Más conocida como Integrated Border Management (IBM).}

Esta institución es un pilar fundamental del objetivo de gestión europea integrada de las fronteras, en adelante GEIF, y es un modelo de cuatro niveles ${ }^{53}$ para el control del acceso que incluye: medidas en terceros países, cooperación con países vecinos, control fronterizo y medidas de control dentro del espacio de libre movimiento, incluyendo el retorno. Todas estas medidas se pueden adoptar en el territorio de la UE, en especial en sus fronteras exteriores e incluso

51 CARRERA, S. BLOCKMANS, S., CASSARINO, J.-P., GROS, D. \& GUILD, E.: The European Border and Coast Guard. Adressing migration and asylum challenges in the Mediterranean?, CEPS, 2017, pág.1

52 "La seguridad es lo que produce el proceso denominado securitización, por el cual se amplían los sujetos susceptibles de sufrir amenazas".

SALAZAR P. ROBINSON E IVONNE YENISSEY R. (2011): "La securitizacion de la seguridad pública: una reflexión necesaria”, pág. 31 en el Cotidiano, puede consultarse el texto en http://www.redalyc.org/pdf/325/32518423004.pdf consultado el 12 de febrero de 2019.

53 Se definía como componente principal en la gestión integral de fronteras en las conclusiones del Consejo de diciembre de 2006.

(C) UNED. Revista de Derecho UNED, núm. 27, 2021 
más allá. En el primer nivel, se enmarcan medidas en terceros países y de forma fundamental en los de origen y tránsito de migración irregular, con los que se intercambia información y actividades consulares relacionadas con visados y formación para los funcionarios respectivos con el fin de atajar la inmigración irregular en origen. En el segundo nivel, las medidas se dirigen a terceros países limítrofes que incluyen la cooperación entre las autoridades de control fronterizo a través de mecanismos normalizados y un intercambio de información adecuado. El tercer nivel se centra en medidas de control de las fronteras exteriores con controles sistemáticos para la detección de actividades criminales de acuerdo con el análisis de riesgos. Por último, un cuarto nivel desarrolla medidas en el interior del espacio Schengen como controles policiales adicionales, cooperación policial y análisis de riesgos con el fin de prevenir y atajar la migración irregular y retorno de migrantes irregulares ${ }^{54}$. La importancia del modelo sigue patente en el nuevo reglamento de la GEFC ${ }^{55}$.

La externalización de la frontera europea se basa de manera principal en los niveles uno y dos del modelo de control de acceso, este tipo de medidas ya las desarrollaba la Agencia, pero no fue hasta el reglamento 1168/2011 cuando se le proporcionó cobertura legal a la cooperación operativa con autoridades de terceros Estados. En el reglamento UE 2016/1624 la GEIF siguió con la ejecución de la promoción de normas en materia de gestión de fronteras y retorno, en el fomento del intercambio de información y su posterior análisis de riesgos y sobre todo en el apoyo a terceros países en su gestión de fronteras e inmigración. El apoyo a terceros países sigue la misma línea de actuación en el nuevo reglamento UE 2019/1896 de la GEFC, pero esta vez delimita y distribuye las funciones de los actores, que quedan plasmadas en un salto cualitativo en lo referente a cooperación con terceros países. Distingue entre relaciones que pueden tener la Agencia y los Estados miembros y además tiene en cuenta la aparición del nuevo cuerpo permanente con su desplie-

54 Decisión n ${ }^{\circ}$ 2/2019 DEL CONSEJO DE ADMINISTRACIÓN FRONTEX de 27 de marzo de 2019, por la que se adopta una estrategia técnica y operativa de gestión europea integrada de las fronteras, pág.14 . Puede consultarse el texto en: https:// frontex.europa.eu/assets/Key_Documents/MB_Decision/2019/MB_Decision_2_2019_ adopting_a_Technical_and_Operational_Strategy_for_European_Integrated_Border_Management.pdf. Consultado el 14 de abril d 2020.

55 Considerando (11): "La gestión europea integrada de las fronteras, basada en el modelo de control de acceso de cuatro niveles, incluye medidas en terceros países como las previstas en el marco de la política común de visados, medidas con terceros países vecinos, medidas de control de las fronteras exteriores, un análisis de riesgos y medidas tomadas dentro del espacio Schengen y medidas en materia de retorno". 
gue fuera del territorio de la Unión para proteger la frontera exterior y la gestión eficaz de la política de migración de la Unión ${ }^{56}$.

Los acuerdos de trabajo han sido, hasta la fecha, el instrumento central de la cooperación de FRONTEX con terceros Estados ${ }^{57}$. Su contenido a lo largo de todos estos años ha seguido una misma estructura en la que se describen los procedimientos para intercambiar información, coordinación de operaciones conjuntas y proyectos piloto, análisis de riesgos y desarrollos técnicos para la gestión de los controles fronterizos, así como la formación en la materia. La firma de un acuerdo de trabajo apareció regulada por primera vez en la Decisión del Consejo de Administración de FRONTEX de 1 de septiembre de 2006. Durante estos años, se ha regulado en los distintos reglamentos el modo en que se han de celebrar. De la negociación se encarga el director ejecutivo con las premisas previas del Consejo de Administración, que en último lugar debe aprobarla. Antes de su aprobación por el Consejo de Administración, debe recibir el visto bueno de la Comisión europea. En la versión 2.0 de la GEFC el texto del artículo 76.4 no hace más que reforzar la idea de la necesidad de un mayor papel del Parlamento en este tipo de procedimientos, que ya se había planteado en el debate académico como manifiestan algunos autores ${ }^{58}$.

Los acuerdos de trabajo son un primer peldaño subido por FRONTEX, que completó una etapa de operaciones que llegaron a su límite operativo. Muchas veces fue y sigue siendo acusada la Agencia del abuso en la utilización de este instrumento administrativo carente de consideración como tratado internacional para arrogarse una personalidad internacional de la que carece. El segundo peldaño lo constituyen las operaciones que exijan el despliegue del cuerpo permanente en un tercer país y que conlleven competencias ejecutivas. En estos casos, no es suficiente un acuerdo de trabajo ya que es necesario un acuerdo sobre el estatuto con ese país (así viene recogido en la nueva normativa ${ }^{59}$ ).

Si analizamos con detalle el nuevo reglamento y en concreto los preceptos sobre esta tarea de FRONTEX, encontramos el primer in-

56 Considerando (87) del reglamento (UE) 2019/1896.

57 FINK, M. "Frontex Working Arrangements: Legitimacy and Human Rights Concerns Regarding

Technical Relationships". Utrecht Journal of International and European Law, $\mathrm{n}^{\circ}$ 28 2012, pp. 20-35. Disponible en: https://doi.org/10.5334/ujiel.be.

58 SPENGEMAN, A. "Upholding the legitimacy of Frontex: European Parliamentary Oversight”. European Security Review, 2013 pp.1-9.

59 Artículo 73.3 del reglamento (UE) 2019/1896. 
dicador de la apuesta por la externalización de las fronteras en el artículo destinado a enumerar los once elementos que conforman la GEIF, y que determina la cooperación con terceros países y con especial importancia en los países vecinos, los países de origen o tránsito de inmigración ilegal ${ }^{60}$. El desarrollo de este elemento se ejecuta a través del artículo 71, que establece las bases de cooperación con terceros países. Designa como sujetos activos a los Estados miembros y a la Agencia para que proporcionen asistencia técnica y operativa a terceros países, siempre con un mismo objetivo de mejora de la GEIF y como límite el respeto al Derecho de la Unión y su acervo, tanto si actúan dentro como fuera del territorio comunitario. Es significativo el cambio de rol que sufre FRONTEX, puesto que pasa de un papel secundario de mero facilitador de cooperación técnica u operativa entre Estados miembros y terceros países, ${ }^{61}$ a actor principal equiparándose en materia de cooperación a los Estados miembros. En este sentido, la Agencia ya no tendrá que depender de las delegaciones de la Unión en terceros países para cooperar y podrá establecer a través de su consejo de administración una antena ${ }^{62}$. Este cambio de rol es tan evidente que el nuevo reglamento distribuye en varios artículos las diferentes relaciones que pueden existir entre actores distinguiendo de forma clara sus potestades.

\section{IV.A.1. Cooperación de Estados miembros con terceros países.}

En primer lugar el art. 72 regula la cooperación entre Estados miembros y terceros países. En esta relación, se habilita a los primeros a una cooperación a nivel operativo que elimina el límite material que se encontraba en el intercambio de información y extiende el límite territorial, ya no solo a la celebración de acuerdos con terceros países vecinos, sino con cualquier tercer país ${ }^{63}$. Sin embargo, los Estados miembros siguen obligados a que esta cooperación operativa con terceros estados, e incluso con otros Estados miembros, sea compatible con las tareas de la Agencia, la cual debe de ser infor-

60 Artículo 3.1 apartado g) del reglamento (UE) 2019/1896.

61 Artículo 54.1 del reglamento (UE) 2016/1624.

62 Artículo 100.1 apartado c). La antena es otra de las novedades que presenta este nuevo reglamento de la GEFC 2.0 en su artículo 60. Se trata de crear un interfaz que desempeñe tareas de coordinación, logística y apoyo, facilitando la cooperación entre diferentes actores inmersos en un fin común. La Agencia mejorará su comunicación con otras agencias, instituciones, Estados miembros de acogida o terceros países.

63 Artículo 72.1 del reglamento (UE) 2019/1896 que sustituye al art. 20.1 del reglamento (UE) 1052/2013 EUROSUR. 
mada de los acuerdos a los que se lleguen ${ }^{64}$. La Unión, a día de hoy fomenta la externalización de las fronteras en todos los frentes posibles mediante acuerdos bilaterales o multilaterales con el mayor número de actores implicados, lo que hace mantener a los Estados miembros un importante poder decisorio en esta materia, muy lejos de la federalización que nos quieren hacer ver.

\section{IV.A.2. Cooperación de la Agencia con terceros países.}

En segundo lugar, se regula la cooperación de la Agencia con terceros países ${ }^{65}$ para desempeñar sus funciones conforme al reglamento. La forma en que se desarrollará la relación entre estos actores ha sido perfilada al detalle, lo que denota un interés en esta forma de gestión de fronteras, que hace prever futuros despliegues del nuevo cuerpo permanente en el exterior de la zona Schengen. Controla circunstancias hasta ahora controvertidas en el ámbito jurídico como, por ejemplo, la validez de los acuerdos de trabajo de FRONTEX. En este sentido, los despliegues operativos que impliquen competencias ejecutivas fuera de territorio Schengen, deberán quedar bajo el auspicio de la celebración de acuerdos de estatuto entre la Unión y el tercer país. Este tipo de acuerdos tiene rango de tratado internacional y por ello necesitan de una tramitación con las máximas garantías y el procedimiento, sólo se puede llevar a cabo con el beneplácito de las instituciones europeas. Sin embargo, la cooperación para la asistencia técnica o el intercambio de información sólo precisará un acuerdo de trabajo. Esta fórmula limita la autonomía de la Agencia que no puede por si misma desplegar en el exterior al cuerpo permanente sin supervisión de las instituciones europeas. La Agencia, respecto del ya de por sí limitado instrumento de los acuerdos de trabajo, se encuentra además con un importante veto que demuestra el poder soberano de los Estados miembros. El representante del Consejo de Administración del Estado miembro limítrofe con el tercer estado que se pretenda celebrar un acuerdo de trabajo, debe votar a favor para que el mismo sea viable ${ }^{66}$.

64 Artículo 7.5 del reglamento (UE) 2019/1896.

65 Artículo 73 del reglamento (UE) 2019/1896.

66 Artículo 100.3 del reglamento (UE) 2019/1896. 


\section{IV.A.2.a). Acuerdos de estatuto}

En la actualidad, la Unión ha celebrado acuerdos de estatuto amparados en el artículo 73.3 del reglamento UE 2019/1896 ${ }^{67}$ con tres países, el primero fue Albania en octubre de 2018, después con Montenegro en octubre de 2019 y por último con Serbia ${ }^{68}$ firmado el 19 de noviembre de 2019 con autorización para la firma mediante decisión del Consejo ${ }^{69}$. Existen otros dos acuerdos similares firmados con Macedonia del Norte en julio de 2018 y con Bosnia Herzegovina en enero de 2019 pendientes aún de aprobación. Como se puede observar, en los últimos dos años se empiezan a celebrar este tipo de acuerdos que muestran un mayor control sobre las actividades operativas de la Agencia fuera del territorio de la Unión, cuando con anterioridad únicamente se firmaban acuerdos de trabajo.

\section{IV.A.2.b). Acuerdos de trabajo}

En cuanto a los acuerdos de trabajo, podemos significar que FRONTEX ha celebrado veinte de ellos ${ }^{70}$ con autoridades de terceros países con especial foco sobre los Balcanes. Este interés, ha provocado un incremento de la cooperación que desemboca en la celebración de acuerdos de estatuto y eleva a ese segundo peldaño del que acabamos de hablar, en el que resulta un trabajo conjunto con una mayor implicación por ambas partes. El fortalecimiento de las fronteras no finaliza aquí, puesto que la Agencia aspira a establecer acuerdos de cooperación con alianzas en el norte de África incluido, por supuesto, Marruecos ${ }^{71}$.

67 En aquel momento basados en el vigente artículo 54.4 del Reglamento (UE) 2016/1624.

68 Puede consultarse el texto en: https://eur-lex.europa.eu/legal-content/ES/TXT/ PDF/?uri=CELEX:32019D0400\&qid=1587492971173\&from=ES. Consultado el 21 de abril de 2020.

69 DECISIÓN (UE) 2019/400 DEL CONSEJO de 22 de enero de 2019.

70 Con Rusia, Ucrania, Moldavia, Georgia, Macedonia, Serbia, Albania, Bosnia Herzegovina, Estados Unidos, Montenegro, Bielorrusia, Canadá, Cabo Verde, Nigeria, Armenia, Turquía, Azerbaiyán, Kosovo, Consejo de Comandantes de Tropas Fronterizas de la CEI y Centro regional MARRI de los Balcanes Occidentales. Puede consultarse el texto en la página oficial de FRONTEX. https://frontex.europa.eu/ about-frontex/key-documents/?category=working-arrangements-with-non-eu-countries. Consultado el 22 de abril de 2020.

71 Puede consultarse el texto en: https://www.elmundo.es/internacional/2019/12/ 06/5de9517621efa0275a8b4594.html. Consultado el 9 de abril de 2020. 
IV.A.3. Relevancia de la cooperación con terceros países ${ }^{72}$.

Cuando en el anterior epígrafe comparaba las tareas ejecutivas de las dos versiones de los reglamentos de la GEFC, el lector habrá podido percatarse que no he realizado referencia alguna al artículo 10.1 y en especial a sus apartados $\left.\mathrm{u})^{73} \mathrm{y} \mathrm{v}\right)^{74}$. El motivo ha podido ser un olvido intencionado que permita introducir esas tareas en esta parte del artículo en la que tendrá mejor acomodo. Ambos apartados no aparecen en el antiguo artículo 8 del reglamento UE 2016/1624 que recogía las tareas de la GEFC, lo que no significa que no estuviera previsto. El antiguo artículo 54 recogía estas dos tareas que ahora en el nuevo reglamento, son elevadas en relevancia al situarlas en el artículo 10 de tareas de la Agencia europea de la Guardia de Fronteras y Costas y su posterior desarrollo normativo por los ya expuestos en este epígrafe artículos 73 y 74. Su nueva localización en ese importante artículo determina la plasmación jurídica de los deseos políticos de desplazar la lucha contra la migración fuera del territorio Schengen.

\section{IV.A.4. Operaciones militares.}

El proceso de externalización, se trata de reforzar con todos los medios disponibles por parte de la Unión incluidas las operaciones militares $^{75}$. Tanto el nuevo texto como el antiguo sólo hacen mención una vez a este tipo de operaciones, pero se ha hecho uso de ellas y, como ejemplo de ello, encontramos la operación "SOPHIAEUNAVFORMED”76 de la Unión Europea en el Mediterráneo central meridional que ha sido sustituida en recientes fechas por la operación "IRINI-EUNAVFORMED”.

72 MARÍN, L. "The Cooperation Betwen Frontex and Third Countries in Information Sharing: Practices, Law and Challenges in Externalizing Border Control Functions", en European Public Law, Vol 26 (1), 2020, pp. 157-180.

${ }_{73}$ "cooperar con terceros países en relación con los ámbitos cubiertos por el presente Reglamento, incluso a través del posible despliegue operativo de equipos de gestión de fronteras en terceros países".

${ }_{74}$ "asistir a los Estados miembros y a terceros países en el contexto de la cooperación técnica y operativa entre ellos en los ámbitos regulados por el presente Reglamento";

75 Considerando (85) del reglamento (UE) 2019/1896.

76 Puede consultarse el texto en: https://www.defensa.gob.es/misiones/en_exterior/actuales/listado/eunavformed.html. Consultado el 22 de abril de 2020.

(C) UNED. Revista de Derecho UNED, núm. 27, 2021 


\section{IV.A.4.a) Operación SOPHIA.}

Esta operación se desarrolló frente a las costas libias para luchar contra las redes de tráfico de personas, prevenir flujos de migración irregular y evitar que muriera más gente en el mar. Por la persistente falta de solidaridad entre los Estados miembros, tuvo que ser suspendida temporalmente el 27 de marzo de 2019 al no existir un acuerdo sobre el desembarco de los inmigrantes rescatados y finalizada con carácter permanente a partir del 31 de marzo de 2020.

Desde que se puso en marcha ${ }^{77}$ el 22 de junio de 2015, se configuró como una respuesta integral de la UE al fenómeno de la migración. Tratando de abordar no solo su componente físico, sino también la raíz de las causas que incluían los conflictos, la pobreza, el cambio climático y la persecución. Unos fines ambiciosos que finalizaban con la implementación de medidas militares de fortalecimiento de las fronteras europeas y olvidaba el resto. Para darle mayor legitimidad, la operación se llevó a cabo bajo el mandato de la Resolución 2240 del Consejo de Seguridad de las Naciones Unidas ${ }^{78}$. La misma, facultaba a los Estados miembros a actuar de forma individual o en el marco de organizaciones regionales, con el fin de utilizar todas las medidas necesarias y enfrentarse a los contrabandistas o traficantes de seres humanos en cumplimiento de las leyes sobre derechos humanos.

Desde un principio, la operación se centró en una competencia que afectaba directamente a dos agencias como FRONTEX y EUROPOL y que no era otra cosa que la desarticulación de las organizaciones criminales que suponen las redes de tráfico de personas humanas en el Mediterráneo Central. Para ello, realizó esfuerzos sistemáticos para identificar, capturar y destruir las embarcaciones utilizadas o sospechosas de ser usadas por los traficantes, de acuerdo con las normas internacionales y al mismo tiempo, contribuyó a dificultar la entrada de migrantes ilegales por otros medios.

En marzo de 2019, el desembarque de personas rescatadas fue uno de los últimos escollos entre los Estados miembros que dejó sin barcos y sólo con efectivos aéreos a la operación que se encaminaba

77 El 18 de mayo, el Consejo aprobó el Concepto de Gestión de la Crisis para una misión militar dentro de la Política Común de Seguridad y Defensa (CSDP) a fin de interrumpir el negocio de las redes de contrabando y tráfico de personas en la Zona Sur del Mediterráneo Central. (Decisión 2015/778 de 18 de mayo de 2015).

78 Resolución 2240 (2015) de mantenimiento de la paz y seguridad internacionales, aprobada por el Consejo de Seguridad en su $7531^{\text {a }}$ sesión, celebrada el 9 de octubre de 2015. S/RES/2240 (2015) 
sin remisión a su final. Tras meses de negociaciones dentro de la Unión, dos de los Estados miembros que más reticencias presentaban fueron Austria y Hungría, denunciando que los rescates marítimos que realizaban los buques de la operación producían un "efecto llamada" para los migrantes que se encontraban frente a las costas de Libia, esta posición fue la que terminó con la operación SOPHIA. Para desbloquear la situación y reactivar una operación similar en la zona, hubo que desplazar hacia el este el emplazamiento en el que operarían los buques para que Austria y Hungría eliminaran sus reticencias y, además la nueva operación no tendría como mandato el rescate de migrantes. Esta solución es cuanto menos discutible, puesto que no podemos olvidar que el Derecho del mar obliga a socorrer a barcos en dificultades, por lo que los barcos que participen tendrán implícita esta misión ${ }^{79}$.

\section{IV.A.4.b) Operación IRINI.}

A resulta de las discusiones entre Estados miembros, la operación naval IRINI, sucederá a la misión SOPHIA en el Mediterráneo central. Para ello el Consejo de la UE aprobó la puesta en marcha de la Operación EUNAVFOR MED IRINI ${ }^{80}$, cuyo mandato se centra en la ejecución del embargo de armas en Libia a través de recursos aéreos, marítimos y satelitales. La duración de la nueva operación se prevé que se extenderá hasta el 31 de marzo de 2021. Su desarrollo se realizará frente a la costa del este de Libia lejos de las rutas usadas por los traficantes de migrantes, como pedían Austria y Hungría. De hecho, la lucha contra los traficantes de migrantes pasa a ser una función secundaria dentro de las actuaciones que se puedan realizar. A partir de ahora, los integrantes de la operación deberán realizar inspecciones de embarcaciones en alta mar frente a la costa de Libia, cuando sean sospechosas de transportar armas o material relacionado desde o hacia el país de acuerdo con la Resolución 2292 (2016) del Consejo de Seguridad de la $\mathrm{ONU}^{81}$. Asimismo, como ta-

79 Convención de las Naciones Unidas sobre Derecho del Mar (CNUDM). Puede consultarse el texto en https://www.boe.es/buscar/doc.php?id=BOE-A-1997-3296, consultado el 10 de abril de 2020.

${ }_{80}$ Puede consultarse el texto en la página oficial del Departamento de Seguridad Nacional del Gobierno de España https:/www.dsn.gob.es/es/actualidad/seguridad-nacional-ultima-hora/uni\%C3\%B3n-europea-libia-\%E2\%80\%93operaci\%C3\%B3n-irini. Consultado el 22 de abril de 2020.

81 Autoriza, por un período de 12 meses, a que se realicen inspecciones en alta mar, frente a las costas de Libia, de buques sobre los que existan motivos para creer

(C) UNED. Revista de Derecho UNED, núm. 27, 2021 
reas secundarias, ${ }^{82}$ podrá supervisar y recopilar información sobre las exportaciones ilícitas de petróleo, contribuir al desarrollo de capacitación de la Guardia Costera y la Armada de Libia, así como la desarticulación del modelo de negocio de las redes de tráfico ilícito y trata de personas mediante la recopilación de información y patrullas aéreas.

IRINI (que significa "paz» en griego), consistirá de manera principal en el aseguramiento del embargo de armas decretado por Naciones Unidas. Este mandato estará sujeto a la estrecha vigilancia de los Estados miembros de la UE, que mantendrán el control político y la dirección estratégica a través del Comité Político y de Seguridad (CPS), bajo la responsabilidad del Consejo y del Alto Representante de la Unión para Asuntos Exteriores y Política de Seguridad.

\section{IV.A.5. Mayor externalización al mismo ritmo.}

De lo expuesto en este apartado tercero, se puede extraer una rápida conclusión: la Unión quiere potenciar aún más si cabe, la externalización de su frontera. El nuevo reglamento, lejos de facultar con nuevas tareas a la Agencia, mantiene las ya existentes, al tiempo que perfila y aclara aquellos aspectos que realizaba y que le han supuesto algún problema operativo o jurídico en el trabajo de campo. Por ello, destaca la escrupulosa distinción entre todas las posibles relaciones que se pueden dar entre actores, y que en la versión 1.0 de la GEFC no se distinguían. Por ejemplo, distingue de forma clara cuando es la Agencia o cuando es un Estado miembro el que negocia con un tercer Estado, qué tipo de instrumento jurídico debe utilizar y el procedimiento a seguir. Con anterioridad, ambos actores eran tratados como uno indisoluble y no se diferenciaban tan claramente sus potestades. Lo importante de verdad para la Unión es fomentar este tipo de acuerdos, procedan del actor que procedan, y por eso se esfuerza en ampliar lo legislado al respecto.

Sin embargo, consideramos que la mayor dificultad que se encontrará la Unión de cara a un estrategia común europea en materia de migración, son los diferentes intereses particulares que presentan los distintos Estados miembros, los cuales se manifiestan en las dife-

que transportan armas o material conexo a Libia o desde su territorio, en violación del embargo de armas. S/RES/2292 (2016).

82 Puede consultarse el texto en la página oficial del Consejo europeo https:// www.consilium.europa.eu/es/press/press-releases/2020/03/31/eu-launches-operationirini-to-enforce-libya-arms-embargo/. Consultado el 22 de abril de 2020. 
rencias políticas, las dificultades para llegar a acuerdos y la falta de solidaridad ante situaciones adversas.

\section{CONCLUSIONES.}

La complicada estructura institucional de la Unión enmascara para los legos en integración europea una lucha de poder entre instituciones supranacionales e intergubernamentales. Este sistema, presenta un abanico de competencias cuyos extremos comprenden desde las exclusivas de la UE (supranacionales), con la consiguiente pérdida total de la soberanía nacional de los veintisiete Estados miembros, hasta el extremo opuesto de competencias de apoyo (intergubernamentales), con un papel testimonial de la Unión. Este reparto nos deja un terreno intermedio en el que se sitúan las competencias compartidas, en el que encontramos un escenario de ambigüedad e indeterminación que dificulta la resolución de los conflictos creados por los intereses divergentes de los actores europeos. Justo en esta zona intermedia, se halla el artículo 4.2 del TFUE con el Espacio de Libertad, Seguridad y Justicia que se encarga de las políticas sobre controles de las fronteras tal como establece el artículo 77 del TFUE.

Uno de los grandes éxitos de la Unión desde su origen, ha sido su ampliación pasando de seis países fundadores a los actuales veintisiete. Lo que en un principio se veía como una fortaleza ha mutado a debilidad debido a la dificultad de responder a las diferentes peticiones de más o menos Europa, y genera una importante dicotomía en la que participan un elevado número de actores.

En este escenario de riesgo para el proceso de construcción europea, FRONTEX debe afrontar los factores contrapuestos que acabamos de mencionar. Así, en primer lugar, tiene que operar en una competencia situada en la zona gris del ordenamiento europeo, que va a generar choques entre el poder institucional supranacional representado por la Comisión y de otra parte la defensa a ultranza de su soberanía por los Estados miembros como poder intergubernamental. De sobra conocida es la dificultad en el diálogo a tres en el ámbito de las competencias compartidas. En segundo lugar, se ha encontrado desde el año 2015 con la mayor crisis migratoria jamás conocida después de la Segunda Guerra Mundial.

Con este panorama y la opinión pública presionando, el poder institucional supranacional se embarcó en la modificación del mandato de FRONTEX, pero cometió toda una serie de errores en el pro- 
ceso de modificación de la normativa (cortoplacismo, políticas de posverdad y populismo punitivo), y trató de ofrecer una sensación de seguridad para los ciudadanos europeos a través de la imagen de Europa fortaleza.

Por todo ello, consideramos que el nuevo reglamento UE 2019/1896 de la GEFC ha utilizado a FRONTEX como una navaja suiza (capaz de desplegar sus herramientas y útiles), que debe solucionar los distintos problemas de seguridad presentes en el territorio Schengen, para ello, la Comisión diseñó un proyecto muy potente avalado con un gran esfuerzo presupuestario, que apostaba por la capacidad de los medios humanos y materiales además de un incremento en tareas ejecutivas. Ese proyecto exigía un paso más de integración y la pérdida de soberanía por parte de los Estados miembros, que con rapidez se opusieron en la cumbre de Salzburgo de septiembre de 2018. Los Estados, encabezados por España e Italia, en lugar de la creación del nuevo cuerpo permanente solicitaban esos fondos para reforzar sus propios cuerpos de guardia de fronteras y costas. En contra de esta defensa de la soberanía nacional se encontraban otros miembros como Alemania o Austria. Al final se impusieron las tesis españolas y el proyecto quedó menguado.

Durante la tramitación legislativa del reglamento se producen modificaciones en la propuesta inicial, que plasman las posiciones discordantes de los actores europeos. De ello resulta un Cuerpo permanente de gran capacidad, pero que carece de tareas ejecutivas que amplíen su espectro anterior. Los Estados miembros imponen su soberanía restringiendo de modo considerable las pretensiones iniciales propuestas por la Comisión. El texto definitivo articula una posibilidad de acción de la Agencia que encuentra su límite en la aquiescencia del Estado miembro, dejando patente dos visiones distintas del futuro europeo y que dificulta gravemente la resolución de los problemas migratorios.

Uno de los aspectos positivos del nuevo reglamento, es la cobertura legal de algunas prácticas que ya desarrollaba la Agencia y que quedan por fin reguladas. Un ejemplo muy significativo es el porte y uso de armas por miembros de la GEFC en otros Estados miembros que ahora se recoge en el Anexo V. También la externalización de las fronteras es potenciada y se disipan las funciones de cada actor individualizando cual debe ser su comportamiento.

Como conclusión final, consideramos que la cuestión principal que debe plantearse la Unión de cara al futuro es el salto definitivo del Espacio de Libertad, Seguridad y Justicia al artículo 3 del TFUE 
como competencia exclusiva. El modelo actual de la GEFC está confinado y agotado por el artículo 4.2 del TFUE. El informe UNISYS del año 2014 establecía tres fases para que FRONTEX fuera un actor clave en la protección de fronteras. Actualmente se encuentra anclada en la segunda fase de transferencia de poderes ejecutivos que ha redondeado con las dos versiones normativas del reglamento de la GEFC, pero que ya no tienen más recorrido que transitar a la tercera fase con una agencia de plenos poderes ejecutivos centralizada en un órgano supranacional. Para ello, se necesita una cesión de soberanía de los Estados miembros y un nuevo encaje de la gestión de fronteras en el reparto competencial europeo. 
